\title{
Moonlighting Proteins Hal3 and Vhs3 Form a Heteromeric PPCDC with Ykl088w in Yeast CoA Biosynthesis
}

\author{
Amparo Ruiz ${ }^{1, \#,+}$, Asier González ${ }^{1,2,+}$, Ivan Muñoz ${ }^{1, \ddagger}$, Raquel Serrano ${ }^{1, \dagger}$, J. Albert Abrie ${ }^{3}$, \\ Erick Strauss $^{3}$ and Joaquín Ariño ${ }^{1,2 *}$ \\ ${ }^{1}$ Departament de Bioquimica i Biologia Molecular, Universitat Autonòma de Barcelona, \\ ${ }^{2}$ Institut de Biotecnologia i Biomedicina, Universitat Autònoma de Barcelona, Spain \\ and \\ ${ }^{3}$ Department of Biochemistry, Stellenbosch University, South Africa
}

*To whom correspondence should be addressed:

Dr. Joaquín Ariño,

Departament de Bioquímica i Biologia Molecular,

Ed. V, Universitat Autònoma de Barcelona,

Bellaterra 08193, Barcelona, Spain,

Phone: 34-93-5812182,

FAX: 34-93-5812006,

E-mail: Joaquin.Arino@UAB.ES

${ }^{+}$Both authors contributed equally to this work.

\# Present address: Columbia University, Departments of Genetics \& Development and Microbiology, New York, USA.

${ }^{\ddagger}$ Present address: MRC Protein Phosphorylation Unit, Sir James Black Centre, University of Dundee, Dundee, Scotland, UK.

${ }^{\dagger}$ Present address: Department of Biological Sciences, Stanford University, Stanford, CA, USA. 


\begin{abstract}
Unlike most other organisms, the essential five-step Coenzyme A biosynthetic pathway has not been fully resolved in yeast. Specifically, the gene(s) encoding the phosphopantothenoylcysteine decarboxylase (PPCDC) activity still remains unidentified. Sequence homology analyses suggest three candidates, namely Ykl088w, Hal3 and Vhs3, as putative PPCDC enzymes in Saccharomyces cerevisiae. Interestingly, Hal3 and Vhs3 have been characterized as negative regulatory subunits of the Ppz1 protein phosphatase. Here we show that YKL088w does not encode a third Ppz1 regulatory subunit, and that the essential roles of Ykl088w and the Hal3/Vhs3 pair are complementary, cannot be interchanged and can be attributed to PPCDC-related functions. We demonstrate that while known eukaryotic PPCDCs are homotrimers, the active yeast enzyme is a heterotrimer which consists of Ykl088w and Hal3/Vhs3 monomers that separately provides two essential catalytic residues. Our results unveil Hal3/Vhs3 as moonlighting proteins, involved in both CoA biosynthesis and protein phosphatase regulation.
\end{abstract}


Coenzyme A (CoA, 1) is a ubiquitous and essential cofactor that is utilized by a wide variety of enzymes in reactions where it mainly acts as a carrier and activator of acyl groups ${ }^{1 \text {, }}$ 2 . The CoA biosynthetic pathway has been elucidated in various diverse species, including eubacteria (Escherichia coli), plants (Arabidopsis thaliana), and mammals (Homo sapiens) ${ }^{1,3}$, ${ }^{4}$. These studies have shown that the pathway is universal and consists of the same five enzymatic transformations in all cases, although some diversity exist among the specific proteins that catalyze certain steps ${ }^{5}$. Nonetheless, bioinformatic approaches allow identifying (with a few exceptions ${ }^{6}$ ) candidate genes encoding the CoA biosynthetic proteins in nearly all organisms, including Saccharomyces cerevisiae.

Interestingly, sequence homology searches suggest three proteins, namely Hal3, Vhs3 and Ykl088w, as candidates that may exhibit phosphopantothenoylcysteine decarboxylase (PPCDC) activity in S. cerevisiae. PPCDC is a flavoprotein that catalyzes the decarboxylation of 4'-phosphopantothenoylcysteine (PPC; 2 ) to yield 4'-phosphopantetheine (PP; 3 ), the third step in CoA biosynthesis. While previous studies on PPCDCs have shown some diversity among these enzymes (e.g. the bacterial variants are usually bifunctional proteins that also have phosphopantothenoylcysteine synthetase (PPCS) activity), they all share a common mechanism and active site architecture ${ }^{7-11}$. Furthermore, all PPCDCs characterized so far are monogenic. While the three PPCDC candidates in S. cerevisiae are indeed very similar (Vhs3 and Ykl088w have 49\% and 28\% sequence identity to the Hal3 protein respectively, see Figure 1a), the identification of Hal3 and Vhs3 as potential PPCDCs is in fact surprising as both proteins have previously been shown to have functions completely unrelated to CoA biosynthesis.

Specifically, Hal3 (also known as Sis2) is a conserved protein originally identified as a halotolerance determinant ${ }^{12}$ and as a high copy-suppressor of the growth defect of cells deficient in the Sit4 protein phosphatase ${ }^{13}$. Both functions have been linked to a Hal3mediated downregulation of the Ser/Thr protein phosphatase Ppz1 ${ }^{14}$, since Hal3 binds to the carboxy-terminal catalytic domain of Ppz1 and strongly inhibits its phosphatase activity ${ }^{15}$. Decreased activity of Ppz1 leads to salt tolerance, compromised cell integrity and accelerated G1/S transition ${ }^{16-21}$. Similarly, VHS3 was identified as a multicopy suppressor of the $G_{1} / S$ cell cycle blockade of a conditional sit4 hal3 mutant ${ }^{22}$. Vhs3 also acts as a negative regulator of Ppz1, albeit less potently than $\mathrm{Hal}^{23}$. In contrast, little is known about YKL088w, which was reported to be an essential gene ${ }^{24}$ that encodes a protein of roughly similar size to Hal3 and Vhs3, sharing a carboxy-terminal acidic tail of about 60 residues with its paralogs (Fig. 1a). 
Previous studies in our laboratory ${ }^{23}$ established that the individually viable vhs3 and hal3 mutations are synthetically lethal, and that this effect cannot be attributed to an excess of Ppz phosphatase activity, thus implying an unknown essential function of the Hal3/Vhs3 pair. This finding, in combination with the proposed essentiality of YKL088w and the sequence similarity of all three proteins with known PPCDC enzymes, prompted us to consider the role(s) of these proteins in CoA biosynthesis. Moreover, we found it especially interesting that the homologue of HAL3 in A. thaliana (AtHal3a), while now known to be the PPCDC protein of this organism, was previously shown to also play a role in halotolerance. In fact, the AtHal3a protein can partially complement the salt-sensitive phenotype of a hal3 yeast mutant ${ }^{25}$ even though it is smaller than Hal3, lacking both its amino-terminal extension and acidic tail (Fig. 1a).

Extensive biochemical and structural studies of AtHal3a showed it to be a homotrimer, with the active sites found at the interfaces of the protomers ${ }^{7,10,26}$. Two key and conserved residues were also identified to be important for its PPCDC-catalyzed reaction: His90 and Cys175 (Fig. 1b) ${ }^{8-10}$. These two residues play different roles in the elucidated PPCDC mechanism $^{7}, 11,27,28$, which takes place in two steps as shown in Figure 1c. The conserved His residue is involved in the first step, the FMN (4)-dependent oxidation of PPC's cysteine moiety, which is followed by the spontaneous decarboxylation of the resulting thioaldehyde 5 to yield an enethiol intermediate (6). The second step entails reduction of this intermediate to 4'-phosphopantetheine 3 and reoxidation of the $\mathrm{FMNH}_{2}$ cofactor, and involves the conserved Cys residue. These residues seem to be a general feature of PPCDC enzymes from nearly all organisms, with the exception of the Archaea ${ }^{29}$. Importantly, although the His residue is conserved in all three candidate yeast PPCDCs, the Cys residue is only conserved in Ykl088w (see Figure 1b). This has led to the hypothesis that Ykl088w most likely fulfills the role of $S$. cerevisiae's PPCDC ${ }^{4}$, although no studies published to date has definitively addressed this question, and the nature of the PPCDC activity in yeast therefore remained unresolved.

We set out to elucidate of the roles of the three putative PPCDC proteins in yeast by experimentally finding answers to the following key questions: 1) Is Ykl088w a third regulatory subunit of the Ppz1 protein phosphatase?; 2) Is the essential role of YKL088w due to its unique function as a PPCDC-encoding gene?; and 3) Is the hal3 vhs3 synthetically lethal phenotype related to CoA biosynthesis in some way?

Our results indicate that both Hal3 and Vhs3 are able to execute disparate functions in yeast cells, acting as regulatory subunits of a specific Ser/Thr protein phosphatase as well as participating in the CoA biosynthetic pathway. In contrast, the essential functions of Ykl088w 
seem to be solely based on its role in CoA biosynthesis. Taken together, our data allow us to present a model for the PPCDC activity in S. cerevisiae (which can be extrapolated to other fungi) based on the formation of an atypical heterotrimeric protein consisting of at least one protomer each of Ykl088w and Hal3/Vhs3. In combination, these results provide a novel and interesting example of multifunctional proteins that also offers a fitting explanation to the questions raised above.

\section{RESULTS}

\section{Ykl088w is not an inhibitor of protein phosphatase Ppz1}

Overexpression of Hal3 or Vhs3 results in clear-cut phenotypes that can be attributed to downregulation of Ppz1 activity ${ }^{15,21,30}$. To evaluate the ability of YKL088w to mimic HAL3 behavior, we transformed wild type and hal3 strains with episomal plasmids carrying the HAL3 and the YKL088w genes expressed from their own promoters. As shown in Figure 2a, high-copy expression of HAL3 increased tolerance to lithium, whereas expression of YKL088w had the opposite effect and resulted in cells less tolerant than those carrying an empty plasmid. Remarkably, overexpression of YKL088w did not have any effect in a hal3 strain. High-level expression of HAL3 aggravates the lytic phenotype of a slt2 MAP kinase mutant, which manifests even at the permissive growth temperature of $28^{\circ} \mathrm{C}^{15}$. This is caused by an inhibition of the Ppz1 activity that promotes increased turgor pressure ${ }^{20}$ and can be prevented by providing osmotic support to the cells. In Figure $2 \mathrm{~b}$ we show that overexpression of $Y K L 088 \mathrm{w}$ in a slt2 mutant, instead of inducing cell lysis, supported vigorous growth of the strain. This is also observed when slt2 cells are challenged with caffeine (7), a compound that enhances the lytic phenotype of this mutant ${ }^{31}$. Interestingly, the effect was lost when the experiment was carried out in a slt2 hal3 strain. Expression of $Y K L 088 w$ was also able to rescue growth of a slt2 strain at the non-permissive temperature $\left(37^{\circ} \mathrm{C}\right)$. Further experiments demonstrated that, in contrast to what is seen for $H A L 3$, overexpression of YKL088w negatively affected growth of a sit4 strain and aggravated the characteristic G1 to S phase transition defect observed in these mutants (Supplementary Fig. 1). In essence, our experiments indicated that overexpression of YKL088w caused effects completely opposite to those expected for a gene encoding a Hal3-like protein.

We subsequently investigated if Ykl088w could bind and inhibit Ppz1, and designed both in vitro and in vivo experiments to this end (see Supplementary Methods). Indeed, affinity purification of a GST-fusion of the carboxyl-terminal half of Ppz1 expressed in E. coli, and of the same GST-fusion and a GST-Ppz1fusion expressed in yeast cells, allows 
recovery of small amounts of Ykl088w (Supplementary Figs. 2a and 2b), confirming the interaction recently described in a large-scale interactomic approach ${ }^{32}$. However, and in contrast with what was found for Hal3 and Vhs3 $3^{15,23}$, binding of Ykl088w to the carboxylterminal half of Ppz1 was not more intense than to the entire protein, suggesting a much weaker interaction. Furthermore, bacterially expressed Ykl088w was completely unable to inhibit Ppz1 activity as measured using p-nitrophenyl phosphate (9) as substrate, even under conditions that allowed nearly full inhibition by Hal3 or Vhs3 (Supplementary Fig. 2c). This was confirmed by a different assay based on the dephosphorylation of Reg1 that was previously used ${ }^{23}$ to demonstrate Hal3 and Vhs3 Ppz1-inhibitory capacities (Supplementary Fig. 2d).

Taken together, the experiments presented above indicate that Ykl088w does not behave as an inhibitor of Ppz1 in the cell and, therefore, does not share this physiological function with Hal3 and Vhs3.

\section{YKL088w and Hal3/Vhs3 are not functionally interchangeable}

A systematic analysis of yeast deletion mutants reported that a YKL088w null strain, in which the entire ORF has been deleted, was unviable ${ }^{24}$. However, the YKL088w ORF lays only 269 bp away from the MIF2/YKL089W stop codon, and MIF2 codes for a kinetochore protein whose mutation is deleterious to the cell ${ }^{33}$. We therefore confirmed the essential nature of YKL088w by disruption of the gene in a diploid strain and overexpression of wild type YKL088w ORF in the heterozygous diploid mutant, followed by sporulation and tetrad analysis (Supplementary Fig. 3).

Given the similarity between Ykl088w and Hal3 or Vhs3 proteins, as well as the lethal effect of the $y k l 088 w$ and hal3 vhs3 mutations, it was reasonable to test whether overexpression of any of these proteins could allow survival of the other deletion mutants. To this end, diploid strains heterozygous for the diverse mutations and their combinations were prepared and transformed with high copy-number plasmids carrying HAL3, VHS3 or YKL088w. Diploids were induced to sporulate and the phenotype of the haploids analyzed by a combination of random spore and tetrad analysis. The results in Table 1 show that high copy-number expression of YKL088w was unable to rescue lethality of the hal3 vhs3 mutant. Similarly, overexpression of HAL3 or VHS3 did not allow rescue of haploid cells bearing the $y k l 088 w$ mutation. The same results were obtained when the HAL3 and YKL088w ORFs were cloned in the episomal plasmid pWS93, which provides strong and constitutive expression from the $A D H 1$ promoter. These results indicate that, in spite of their structural similarity, the 
essential function of YKL088w cannot be replaced by HAL3 or VHS3 and that the HAL3/VHS3 pair provides vital functions that cannot be fulfilled by high levels of Ykl088w. Our repeated but unsuccessful attempts to isolate high-copy suppressors of both the $y k 1088 \mathrm{w}$ and the hal3 vhs3 mutations further suggests that the $S$. cerevisiae genome does not encode any protein able to functionally replace Ykl088w or the pair Hal3/Vhs3, even when overexpressed.

\section{Ykl088w and Hal3/Vhs3 functions relate to CoA biosynthesis}

To address whether or not the essential functions of Ykl088w and Hal3/Vhs3 could be related to CoA biosynthesis and more specifically as proteins with PPCDC activity, we reasoned that, if so, lethality would be suppressed by bona fide PPCDC enzymes. Therefore, we expressed AtHal3a as well as the human and mouse homologs in heterozygous diploid $y k l 088 w$, hal3 vhs3 and triple mutant strains, and searched for haploid cells carrying the indicated mutations (Table 1). In all cases ykl088w, hal3 vhs3, or even triple mutants were recovered as long as they were expressing the heterologous protein. The same result was obtained by expressing the E. coli $d f p$ gene, which encodes both PPCS and PPCDC activities 8, 34 . These results strongly suggest that the essential Ykl088w and Hal3/Vhs3 functions are involved in the PPCDC activity of CoA biosynthesis.

\section{Reconstitution of yeast PPCDC activity in vitro}

To determine whether the yeast proteins had any PPCDC activity in vitro the Hal3, Vhs3 and Ykl088w proteins were expressed in E. coli as GST-fusion proteins and purified. The GST-tags were subsequently removed before using an established coupled assay (and the human PPCDC enzyme as positive control) ${ }^{11}$ to verify their activity. Each protein was assayed individually, as well in all possible combinations to determine potential synergistic effects. The results, summarized in Figure 3a, indicate that while none of the individual proteins displayed any significant PPCDC activity, combinations of Hal3/Ykl088w and Hal3/Vhs3/Ykl088w were active. Moreover, since addition of Vhs3 to Hal3/Ykl088w (to give the Vhs3/Hal3/Ykl088w combination) increases the activity by 20\%, Vhs3 must contribute to the catalytic process in some manner. Interestingly, the Vhs3/Ykl088w combination exhibited only very low levels of activity.

Taken together, the in vitro activity assay results support the conclusions derived from the genetic complementation studies, and strongly suggest that PPCDC in yeast requires at least Hal3 and Ykl088w to be catalytically active. However, since similar results would be obtained whether the proteins were directly involved in the enzymatic transformation or 
merely activated one protein for PPCDC activity, we set out to clarify their exact role(s) through mutational analysis of previously identified conserved PPCDC active site residues.

\section{Mutagenesis analysis of conserved residues in yeast PPCDCs}

To evaluate the functional relevance of the two conserved catalytic residues of PPCDC (His90 and Cys175 in AtHal3a) in the yeast proteins, the conserved His residues of Hal3, Vhs3 and Ykl088w and the conserved Cys of Ykl088w were mutated to Ala and Ser respectively. The mutated versions were tested for their ability to rescue either the hal3 vhs3 or $y k l 088 w$ mutation in all cases where the native proteins were shown to rescue lethality. The results (Table 1) show that neither Hal3 H378A nor Vhs3 H459A was able to rescue synthetic lethality in the hal3 vhs3 strain, highlighting the importance of the conserved His residue in these proteins. In contrast, Ykl088w H391A allowed survival of the $y k l 088 w$ strain, indicating that the conserved His is not functionally relevant in this context. On the other hand, the results do show that-Ykl088w's Cys478 is essential, since its C478S mutant was not able to rescue the $y k l 088 w$ deletion.

Similar experiments were performed using the plant and human PPCDC proteins expressed from the constitutive PMA1 promoter. Table 1 shows that the H90N mutation in AtHal3a rendered the protein unable to rescue a hal3 vhs3 mutant, while allowing growth in the $y k l 088 w$ background. Conversely, the C175S mutation produced a protein able to rescue the hal3 vhs3 strain, but not-the $y k l 088 w$ deletion mutant. Testing the equivalent C173S mutation in the human PPCDC (HsCoaC) gave similar results. Finally, none of the mutated versions was able to rescue a triple ykl088w hal3 vhs3 mutant strain.

These results allow two relevant conclusions to be drawn: 1) the conserved His determines the essential function only of the Hal3/Vhs3 pair, but not of Ykl088w, indicating that its His391, while conserved, is not required for activity; 2) the conserved Cys of Ykl088w is essential. Considering the putative roles of these residues in the two steps of the PPCDCcatalyzed reaction (Fig. 1c), these conclusions indicate that in yeast either Hal3 or Vhs3 is required to perform the first step, and Ykl088w to complete its second step. An analysis of known PPCDC structures suggests that such a requirement could be satisfied in an elegant fashion by considering a heteromeric model for the yeast PPCDC enzyme.

\section{Proposing a structural model for yeast PPCDC}

The crystal structure of AtHal3a shows that the active protein consists of a homotrimeric assembly where each monomer participates in two separate catalytic sites 
formed at its opposite interfaces (Fig. 3b). For each active site the essential His residue contained in motif II (Fig. 1b) is found on one protomer, while the essential Cys is contained in the substrate recognition clamp (motif IV) of the adjacent molecule. Using the homotrimeric AtHal3a structure as reference, a model of a heterotrimeric yeast PPCDC consisting of one molecule each of Hal3, Vhs3 and Ykl088w can be constructed (Fig. 3c and Supplementary Fig. 4). This model shows that instead of three, such a heterotrimeric protein will only have one single functional active site that contains both the required His (donated by either Hal3 or Vhs3) and Cys (donated by Ykl088w) residues. Such a model accounts for the complementation experiments described above, and explains why it has not been possible to associate the PPCDC activity in yeast with a single protein. However, we must point out that based on this analysis, any heteromer (either dimers or trimers) consisting of at least one Ykl088w protomer and either Hal3 or Vhs3 would also be active in the manner described above. We therefore set out to determine the oligomeric nature of the native yeast PPCDC protein through binding and cross-linking experiments.

\section{Confirming the formation of a heterotrimer in vitro}

Our heteromeric model for the active PPCDC in yeast is supported by previous findings of several high-throughput interactomics experiments that showed the interaction of bait Ykl088w with both Vhs3 and Hal3 ${ }^{35-37}$. As these experiments do not distinguish between direct and indirect interactions, we incubated Hal3 and Vhs3 (after removal of their GST-tags) with GST-Ykl088w bound to glutathione-agarose beads. The beads were extensively washed, followed by treatment with PreScission protease to release Ykl088w and any proteins bound to it. As shown in Figure 4a, Vhs3 and Hal3 co-eluted with Ykl088w, indicating that Ykl088w directly interacts with both Vhs3 and Hal3.The eluted protein mixture was subsequently treated with glutaraldehyde to covalently cross-link interacting proteins. Figure 4a shows that cross-linking results in the disappearance of the individual proteins and in the formation of protein species which migrate to a position corresponding to molecular masses in the range of 210-240 kDa. Considering that the calculated combined molecular mass of Hal3, Vhs3 and Ykl088w is 211 kDa, this provides strong evidence that Hal3, Vhs3 and Ykl088w combine to form a trimeric structure in vitro.

\section{Stoichiometry of the active yeast PPCDC complex}

Although the cross-linking experiments strongly suggest the formation of a heterotrimer in vitro, the activity analyses showed that combinations of Hal3 and Ykl088w 
have PPCDC activity. It is therefore conceivable that a minimal active PPCDC protein can be constituted by joining these proteins in a dimeric complex. To determine if this is indeed the case, or if the formation of a trimer is a requirement for activity, we compared the activity of mixtures of varying amounts of Hal3 and Ykl088w with a mixture of Hal3, Vhs3 and Ykl088w, using a 1:1 mixture of Hal3 and Ykl088w (0.5 $\mu$ g each) as reference point. The results (Fig. 4b) show that, as expected for trimer formation, addition of Hal3 beyond a 2:1 complex has no further effect on activity. However, activity of the Hal3/Ykl088w complex does seem to increase with each corresponding addition of more Ykl088w. While we currently cannot provide a satisfactory explanation for this behavior, it does confirm that Ykl088w is the limiting factor for PPCDC activity. Finally, since the Hal3/Vhs3/Ykl088w mixture has an activity higher than the 1:1 Hal3/Ykl088w mixture (in fact, comparable to that of the 2:1 Hal3/Ykl088w mixture), Vhs3 must also take part in the formation of an active heteromeric yeast PPCDC protein, and should be able to do so even in the absence of Hal3. Indeed, increasing the ratio of Vhs3 to Ykl088w from 1:1 (data in Fig. 3a) to 2:1 (Fig. 4b) significantly increases the activity of this complex.

\section{Activity of yeast PPCDCs with mutated conserved residues}

To demonstrate that all three yeast proteins are directly involved in the PPCDC activity we expressed the Hal3 H378A, Vhs3 H459A and Ykl088w C478S mutants as GSTfusion proteins in E. coli, and purified them as for the native proteins. The activity of the two most active combinations of proteins, i.e. Hal3/Ykl088w and Hal3/Vhs3/Ykl088w, was subsequently assayed, and compared with the same combinations in which one of its members is replaced with its mutant version. The results, summarized in Figure 4c (closed bars), shows that - as expected - any combination containing Hal3 without an active His, or Ykl088w without an active Cys residue displays no significant activity. Furthermore, replacing either Hal3 or Vhs3 with its mutated version in the Hal3/Vhs3/Ykl088w complex also reduces its activity, although the effect is more pronounced when Hal3 is substituted.

Importantly, according to our model, complexes containing the Ykl088w C478S mutant should still be able to catalyze at least the first half of the PPCDC reaction during which $\mathrm{CO}_{2}$ is evolved (see Fig. 1c). Using a $\mathrm{CO}_{2}$-release assay (Fig. 4c, open bars) we demonstrate that the mutant does indeed still catalyze the oxidative decarboxylation of PPCDC, whereas the His378A Hal3 mutant does not. Interestingly, using this assay there is little difference between the activities of the Hal3/Ykl088w and Vhs3/Ykl088w complexes, which may suggest that the reduced activity of the latter complex in the overall reaction is 
related to the stability of the complex, since premature dissociation of the complex would prevent the reduction of the intermediate to complete the reaction.

Taken together, these results provide further evidence that all three proteins are directly involved in the PPCDC-catalyzed reaction, with Hal3/Vhs3 and Ykl088w combining in heterotrimeric complex(es) in which they each respectively provides the particular active site residue required by each of the two PPCDC half-reactions.

\section{Engineering of a homotrimeric S. cerevisiae PPCDC}

With a view to obtaining a functional homotrimeric yeast PPCDC protein, we set out to restore the degenerate catalytic motifs of one of the yeast PPCDC protomers to full catalytic functionality. To this end we substituted Asn466 in Hal3 with a Cys, but found that the resulting protein was still unable to rescue $y k l 088 w$ lethality (data not shown). Since His391 of Ykl088w is not essential in spite of being conserved, we considered whether the nearby sequence insertion (see Fig. 5a) had any effect on the local context of this residue. To test this hypothesis, we replaced the original AWVFDAVNKNDTSLSLNLILㅍHE sequence in Ykl088w by the corresponding EWSSWNKIGDPVL플 sequence from Hal3. The resulting chimera, Ykl088wHis ${ }^{\text {Rep }}$ (for His replacement), was expressed from the YKL088w promoter in ykl088w and hal3 vhs3 strains, as well as in the triple mutant. As predicted, this hybrid protein was not only able to rescue the lethality of the $y k l 088 w$ strain, but also that of the hal3 vhs3 and hal3 vhs3 ykl088w mutants. Remarkably, rescue was achieved even when the construct was expressed from a centromeric plasmid, suggesting that its biological activity is similar to that of the native PPCDC. These results indicate that Ykl088wHis ${ }^{\text {Rep }}$ behaves as a homomeric PPCDC. Moreover, expression of Ykl088wHis ${ }^{\text {Rep }}$ in the temperature sensitive

$d f p-707^{\text {ts }}$ E. coli strain ${ }^{7,38,39}$, in contrast to that of wild-type Ykl088w or Hal3, supported growth at the restrictive temperature of $37^{\circ} \mathrm{C}$ (Fig. 5b).

We also measured the in vitro activity of Ykl088wHis ${ }^{\text {Rep }}$ prepared and purified as for the other proteins. Gratifyingly, the chimeric protein did indeed show activity, although only at high protein concentrations (Fig. 5c). However, addition of either Hal3 or Hal3 and Vhs3 to the protein mixtures increased activity markedly, suggesting that the low activity of the Ykl088wHis ${ }^{\text {Rep }}$ protein is probably due to its reduced ability to form homomeric complexes.

\section{Exploring fungal genomes for putative PPCDC enzymes}

The proposed heteromeric PPCDC model with interdependent monomers in $S$. cerevisiae, which is clearly different from the homotrimeric PPCDC found in A. thaliana and 
most other eukaryotes, raised the question of whether the S. cerevisiae PPCDC is an exception among fungal enzymes. A query of various fungal protein sequence sets with Ykl088w and Hal3 yielded 43 hits in 27 different yeast species (Fig. 6). Three different scenarios could be distinguished: in the first, organisms (such as S. cerevisiae) contain three putative PPCDC-encoding genes similar to Hal3, Vhs3 and Ykl088w. In the second scenario, represented by eight Hemiascomycetes included in the search, only two such genes can be identified. Finally, the Archyascomycete S. pombe and most of the Euascomycetes and Basidiomycetes have a single putative PPCDC-encoding gene, with the exception of Neurospora crassa and Cryptococcus neoformans, which both have duplicate putative PPCDC-encoding genes.

The Hemiascomycetous sequences can be divided into Ykl088w-like (Group1 in Figure 6) and Hal3/Vhs3-like classes (Group 2). All members of Group1 lack the conserved His residue of motif II (Fig. 1b) but do have a conserved Cys residue in the substrate recognition clamp (motif IV) as well as a canonical PXMNXXMW motif (motif III) containing the conserved Asn that participates in decarboxylation ${ }^{9}$. In contrast, all proteins in Group 2 lack these Cys and Asn residues, although they maintain the conserved His embedded in a sequence context similar to that of AtHal3a. The non-hemiascomycetous fungal sequences (Group 3) share all three these signature elements with AtHal3a and human PPCDC. These observations indicate that the phylogenetic spread of distinct, functionally interdependent Ykl088w-like and Hal3/Vhs3-like PPCDC lines largely overlaps with the phylum Hemiascomycetes. S. cerevisiae, Candida glabrata and Vanderwaltozyma polyspora each possess two representatives of the Hal3/Vhs3-like group probably as remnants of the same relatively recent genome duplication event ${ }^{40}$ that occurred shortly before the divergence of the Saccharomyces and Vanderwaltozyma line.

\section{DISCUSSION}

Hal3, Vhs3 and Ykl088w are three related proteins encoded by the yeast genome. Previous work has demonstrated that Hal3 and Vhs3 act in yeast cells as inhibitory subunits of the Ppz1 and Ppz2 protein phosphatases, thus controlling a variety of cell functions such as saline tolerance or cell cycle progression ${ }^{15,18,21,23,41}$. Here we demonstrate that Ykl088w does not play a physiological role as regulatory subunit of Ppz1, as it shows only residual Ppz1 binding capacity and does not affect the phosphatase activity in vitro. This may be explained by the fact that Ykl088w does not conserve several residues found in Hal3 to be important for efficient Ppz1 binding ${ }^{41}$ (see Fig. 1b). In addition, the inability of Ykl088w to inhibit Ppz1 
can be explained by the observation that a conserved Asn in Hal3 (N466) and Vhs3 (N547), which is relevant for Ppz1 inhibition ${ }^{41}$, is exchanged for the catalytically essential Cys478 in Ykl088w. The phenotypes observed from the overexpression of the Ykl088w protein, which are opposite to those observed for Hal3 overexpression, can therefore be explained by the fact that Ykl088w retains some Ppz1 binding capacity without affecting its phosphatase activity. It is conceivable that the excess of Ykl088w produced in the cell by a large increase in the gene copy number would displace native Hal3 from Ppz1, thus mimicking a situation of absence of Hal3 (i.e. a hal3 mutant). This is supported by the observation that the anti-Hal3 effects disappear in hal3 mutants (Fig. 2a,b).

By a combination of genetic and biochemical approaches we showed here that the essential function of Ykl088w is related to its role in CoA biosynthesis, and specifically to PPCDC activity. More importantly, we also demonstrate that the synthetic lethality of the hal3 vhs3 mutation ${ }^{23}$ is also a result of disruption of CoA biosynthesis at the PPCDC level. Our data indicates that, in contrast with the homotrimeric structure defined for PPCDC in plants and mammals, in the yeast $S$. cerevisiae PPCDC is most probably a heterotrimer composed of at least one subunit of Ykl088w and Hal3/Vhs3. Furthermore, in vitro activity analysis suggests that the most active combinations consist of Ykl088w and Hal3 on their own, or together with Vhs3. In the latter case two potential heteromeric complexes could be formed: one as depicted in Figure 3c, and the other with Vhs3 and Hal3 transposed with Vhs3 providing the catalytic His residue. Our data suggest that the latter complex has a much lower activity. Therefore, S. cerevisiae's PPCDC is not a monogenic enzyme, but instead consist of at least two different gene products that are not functionally exchangeable. Previous work has suggested the existence of a 375-400 kDa CoA biosynthesizing complex in S. cerevisiae that would involve an alternative pathway for the biosynthesis of CoA (with a decarboxylase enzyme acting on 5'-ADP-4'-pantothenoylcysteine (10) to form dephospho-CoA (11) $)^{42,43}$. In this regard, we wish to stress that: 1) In spite of the many reported genome-wide experiments to identify protein-protein interactions in S. cerevisiae, no interaction has been described between any of the putative CoA biosynthetic proteins (except for interactions of Ykl088w with Hal3 and Vhs3), suggesting that the heteromeric PPCDC described here is not related to the complex mentioned above. 2) The essential nature of the three proposed components of the PPCDC protein in S. cerevisiae indicates that its requirement for PPCDC activity is absolute and implies that, if it exists, the alternative pathway would not be physiologically relevant. This notion is further supported by our repeated failure to isolate high-copy suppressors of both the $y k l 088 w$ and the hal3 vhs3 mutations, as well as by the observation 
that all genes encoding the additional enzymes composing the classical pathway from pantothenate to CoA are also essential ${ }^{44}$.

From the comparative sequence analysis presented above (Fig. 6), it is clear that duplications of the classical eukaryotic PPCDC-encoding gene are not infrequent, although most of the resulting gene products retain the complete functionality of the ancestral PPCDC as judged from signature sequence elements. In Hemicascomycetes, however, this duplication was followed by the specialization and consequently the interdependence of the descendent lines. It is fair to assume that this cost must have been compensated for by some benefit. It is tempting to speculate that a functional connection exists between the Ppz1 phosphatasemediated role of Hal3 on the energy-requiring processes of potassium uptake and sodium

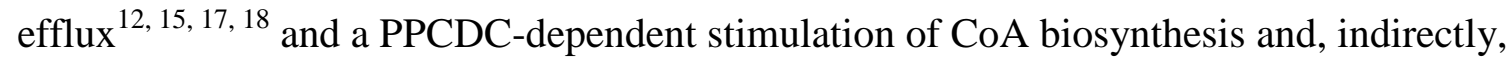
oxidative ATP production. Such an advantage would promote the strengthening of a serendipitously acquired affinity of ancestral Hal3/Vhs3 for Ppz1, even at the cost of an incremental degeneration of its Cys-centered subsite (which overlaps with the Hal3 region implicated in the binding and inhibition of $\mathrm{Ppz} 1^{41}$ ).

Taken together, our results unambiguously map the essential role of Ykl088w in yeast to the PPCDC activity of CoA biosynthesis, and in agreement with a very recent report ${ }^{44}$, we suggest that the corresponding gene's name be changed to CAB3 (for $\underline{\text { Coenzyme } \underline{\mathrm{A}}}$ Biosynthesis) to indicate this fact. Furthermore, the case presented here may also illustrate a crosstalk between regulatory (Ppz1 inhibition) and metabolic pathways (CoA biosynthesis) mediated by the Hal3 and Vhs3 proteins, which would constitute another remarkable example of moonlighting proteins in yeast ${ }^{45}$. 


\section{METHODS}

\section{General}

All chemicals were purchased from Aldrich or Sigma Chemical Co. (unless otherwise indicated) and were used without further purification. Microplate-based enzyme activity measurements were done in either a Multiskan Ascent or Varioskan microplate-based UV spectrophotometer (both from ThermoLabsystems).

\section{Yeast Strains and Growth Conditions}

Yeast cells were grown at $28^{\circ} \mathrm{C}$ in YPD medium (10 g/liter yeast extract, $20 \mathrm{~g} / \mathrm{liter}$ peptone, and $20 \mathrm{~g} /$ /iter dextrose) or, when indicated, in synthetic minimal (SD) medium ${ }^{46}$. Sporulation , tetrad dissection and random spore analysis were done as previously described 46, 47 . Yeast strains used in this study are described in Supplementary Table 1.

\section{Gene Disruption and Plasmid Construction}

Disruption of YKL088w with the kanMX marker was accomplished by the short flanking gene replacement technique using 5'- and 3'-YKL088w_kanMX primers (Supplementary Table 2). The VHS3 gene was disrupted with the kanMX or the nat1 marker as previously described ${ }^{23}$.

Plasmids YEp195-HAL3, YEp195-VHS3, YEp195-HAL3_H378A, YEp195VHS3_H459A, pGEX-HAL3, pGEX-VHS3 and pGEX-PPZ1 were previously described ${ }^{23}$. High-copy expression of YKL088w was achieved by inserting a 3139-bp sequence (starting from an artificial EcoRI site included at position -773 from the ATG codon of YKL088w and ending at the EcoRI site located 647-bp after the stop codon) at the EcoRI site of plasmids YEplac195 (URA3 marker), YEplac181 (LEU2 marker), or YEplac112 (TRP1 marker). An identical cloning strategy using plasmid YCplac111 (LEU2 marker) allowed low copy expression of this gene. Construction of other relevant plasmids is described in Supplementary Methods. All plasmids used in this work are listed in Supplementary Table 3.

\section{Cross-linking experiments}

The interaction of Hal3 and Vhs3 with Ykl088w was assessed in vitro as follows. GST-Ykl088w, GST-Hal3 and GST-Vhs3 constructs (see above) were introduced into E. coli strain BL21(DE3) RIL (Stratagene). GST-Hal3 and GST-Vhs3 were expressed as described ${ }^{23}$. For GST-Ykl088w expression E. coli cultures were grown at $28^{\circ} \mathrm{C}$ and IPTG concentration reduced to $0.1 \mathrm{mM}$. Purification using glutathione-agarose beads (GE Healthcare) was 
performed as described ${ }^{48}$, except that $50 \mathrm{mM} \mathrm{NaH}_{2} \mathrm{PO}_{4}$ (pH 7.0) was used instead of Tris-HCl buffer. GST-Hal3 and GST-Vhs3 containing beads were treated with PreScission protease (GE Healthcare) to remove the GST moiety as recommended by the manufacturers, except that $50 \mathrm{mM} \mathrm{NaH}_{2} \mathrm{PO}_{4}(\mathrm{pH} 7.0$ ) was used as buffer and $10 \%$ glycerol was added to the eluted samples. Fifty $\mu \mathrm{l}$ of beads containing approximately $10 \mu \mathrm{g}$ of GST-Ykl088w were mixed with $45 \mu \mathrm{l}$ of Hal3 $(15 \mu \mathrm{g})$ and $60 \mu \mathrm{l}$ of Vhs3 $(15 \mu \mathrm{g})$ and incubated at $24{ }^{\circ} \mathrm{C}$ for $40 \mathrm{~min}$. Samples were centrifuged and the supernatant removed. Beads resuspended in $200 \mu \mathrm{l}$ of buffer (50 $\mathrm{mM} \mathrm{NaH} \mathrm{PO}_{4} \mathrm{pH}$ 7.0, $150 \mathrm{mM} \mathrm{NaCl}, 10 \%$ glycerol and 0.1\% Triton X-100), transferred to MultiScreen filter plates (Millipore) and extensively washed with the resuspension buffer. Beads were then resuspended in $200 \mu \mathrm{l}$ of washing buffer and one fifth of the volume was set aside to monitor binding of Hal3 and Vhs3 to GST-Ykl088w. The rest was centrifuged and the beads (about $40 \mu \mathrm{l}$ ) were resuspended in $50 \mu \mathrm{l}$ of PreScission buffer and incubated for $7 \mathrm{~h}$ at $4{ }^{\circ} \mathrm{C}$ with 4 units of the protease. The mixture was transferred again to MultiScreen filter plates and the eluate recovered by centrifugation. Thirty $\mu \mathrm{l}$ of the eluate was made $0.005 \%$ glutaraldehyde by adding $3.3 \mu \mathrm{l}$ of a $0.05 \%$ stock solution and incubated for $20 \mathrm{~min}$ at $24{ }^{\circ} \mathrm{C}$.

The cross-linking reaction was stopped by incubation with $2 \mu \mathrm{l}$ of $1 \mathrm{M}$ Tris-HCl pH 7.5 for 5 min. An equivalent cross-link reaction was carried out with $20 \mu \mathrm{l}$ of a $0.1 \mu \mathrm{g} / \mu \mathrm{l}$ solution of bovine serum albumin (BSA) as negative control. Samples received the appropriate volume of $4 \times$ sample buffer and were subjected to SDS-PAGE in 6\% polyacrylamide gels.

\section{Complementation test with a $d f p$ mutant strain of $E$. coli}

The constructs expressing the human PPCDC or the yeast genes HAL3, VHS3, YKLO88w and a version of the latter with the PPC binding motif of HAL3 (YKL088w_His ${ }^{\text {Rep }}$ ) were introduced into the E. coli temperature-sensitive mutant strain BW369 ${ }^{38}$. Transformants were cultured on LB plates supplemented with ampicillin at $30^{\circ} \mathrm{C}$ or $37^{\circ} \mathrm{C}$ for $24 \mathrm{~h}$ and examined for complementation of the $d f p$ mutation.

\section{PPCDC activity assays}

PPCDC activity based on the formation of PP (3) was assayed as described previously ${ }^{11}$ with minor modifications. Assay mixtures $(150 \mu \mathrm{l})$ contained $60 \mu \mathrm{l}$ pyrophosphate reagent, $0.5 \mathrm{mM}$ PPC (synthesized as described), 2.0 mM ATP, $1.0 \mathrm{mM}$ DTT, $10 \mathrm{mM} \mathrm{MgCl}_{2}, 20 \mathrm{mM} \mathrm{KCl}, \sim 1.5 \mu \mathrm{M} 6 \times$ His-EcCoaD and the putative PPCDC protein or proteins in $50 \mathrm{mM}$ Tris-HCl buffer ( $\mathrm{pH}$ 7.8). The PPCDC proteins were loaded into the 
individual wells of a 96-well microplate, followed by a short incubation period at $37^{\circ} \mathrm{C}$. The reaction was subsequently initiated by addition of the rest of the assay mixture at $37^{\circ} \mathrm{C}$, and monitored by following the changes in absorbance at $340 \mathrm{~nm}$.

PPCDC activity based on $\mathrm{CO}_{2}$-formation (i.e. the first PPCDC half-reaction) was determined essentially as described previously ${ }^{49}$ with minor modifications. Assay mixtures

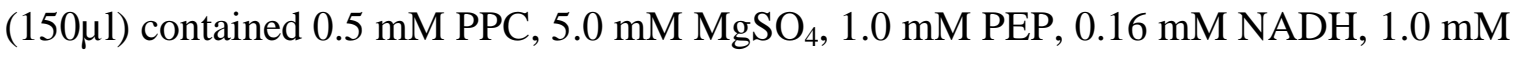
DTT, 1.8 U malate dehydrogenase, 5.5 U lactate dehydrogenase, 0.375 U PEP carboxylase and the putative PPCDCs in $50 \mathrm{mM}$ Tris- $\mathrm{HCl}$ (pH 7.6). The PPCDC proteins were loaded into the individual wells of a 96-well microplate, followed by a short incubation period at $37^{\circ} \mathrm{C}$. The reaction was subsequently initiated by addition of the rest of the assay mixture at $37^{\circ} \mathrm{C}$, and monitored by following the changes in absorbance at $340 \mathrm{~nm}$.

The amount of PPCDC in each assay depended on the experiment; for details see the Supplementary Methods. Activities were measured in either duplicate or triplicate as indicated in the legends to the figures. In all cases the determined activities were corrected for an average measured background rate and were normalized to the activity of the Hal3/Vhs3/Ykl088w mixture (set at 100\%) to allow comparison between experiments. Details of the statistical analysis of the data are provided in the Supplementary Methods. 


\section{ACKNOWLEDGEMENTS}

Thanks are given to B. Weiss for E. coli strain BW369, to A. Osterman for the human PPCDC cDNA and to R. Serrano and P. Sanz for diverse constructs and plasmids. We thank H.

Ceulemans, E. Simón, A. Barceló and A. Vivó for their contribution in different phases of the project. The excellent technical assistance of Anna Vilalta, María Jesús Álvarez and Montserrat Robledo is acknowledged. This work was supported by grant BFU2005-06388C4-04-BMC and BFU2008-04188-C03-01 to J.A. (Ministerio de Educación y Ciencia, Spain and Fondo Europeo de Desarrollo Regional) and grant FA2007041600013 to E.S. (National Research Foundation, South Africa). J.A. is recipient of an "Ajut de Suport a les Activitats dels Grups de Recerca" (2009SGR-1091, Generalitat de Catalunya). A. G. was recipient of a fellowship from the Spanish Ministry of Education and Science. J.A and E.S. are recipients of a Spain/South Africa Research Cooperation grant (HS2007-0022) from the National Research Foundation (South Africa) and the Ministerio de Educación y Ciencia (Spain).

J. A. dedicates this paper to the memory of his father.

\section{COMPETING FINANCIAL INTERESTS STATEMENT}

The authors declare that they have no competing financial interests. 


\section{FIGURE LEGENDS}

Figure 1. Comparison of known and putative PPCDC proteins, and the reaction they catalyze. (a) Cartoon comparing A. thaliana AtHal3a with the S. cerevisiae Hal3, Vhs3 and Ykl088w proteins, with the core PPCDC domains shown in gold, the N-terminal extensions in blue, and the C-terminal acidic tails in pink (uncolored sections denote sequence insertions specific to the protein). Numerical values indicate the \% sequence identity with the $S$. cerevisiae Hal3 protein. The location of the four consensus PPCDC functional motifs (labeled I to IV according to Ref. 7) in each protein is also shown; a light grey label indicates that the motif's sequence deviates from the standard pattern. (b) Alignment of the core PPCDC domain of AtHal3a with those of Hal3, Vhs3 and Ykl088w, highlighting the sequences of the consensus motifs (green background). Residues relevant for PPCDC activity, i.e. the conserved His of motif II (AtHal3a H90), the Asn of motif III (AtHal3a N142) and the Cys of motif IV (AtHal3a C175) are in boldface on a yellow background. Asterisks denote residues found to be necessary in Hal3 for binding or/and inhibition of $\mathrm{Ppz}^{41}$. (c) The reaction catalyzed by the PPCDC enzyme proceeds in two steps: the first step involves the flavin-dependent oxidation of PPC (2) to the thioaldehyde intermediate (5) and has been shown to require the conserved His residue. After the intermediate spontaneously decarboxylates, the conserved Cys residue mediates the second step: the reduction of the enethiolate (6) to give the reaction product 4'phosphopantetheine (3), and oxidized flavin.

Figure 2. Functional characterization of $Y K L 088 w$ as a putative regulatory subunit of Ppz1. (a) Wild type strain JA100 and its hal3 derivative JA104 were transformed with the highcopy plasmid YEplac195 (YEp195) or the same plasmid carrying the HAL3 (YEp-HAL3) or YKL088w (YEp-YKL088w) genes. Cells were spotted on YPD plates containing the indicated concentrations of $\mathrm{LiCl}$ and growth monitored after $48 \mathrm{~h}$ at $28^{\circ} \mathrm{C}$. (b) Strains JC10 (slt2) or EDN101 (slt2 hal3) were transformed as above and grown for $50 \mathrm{~h}$ on YPD plates supplemented as indicated at $28{ }^{\circ} \mathrm{C}$ or $37^{\circ} \mathrm{C}$. Caffeine (7) concentration was $1 \mathrm{mM}$ for slt2 cells and $7 \mathrm{mM}$ for slt2 hal3 cells. The concentration of sorbitol (8), employed as osmotic cushion, was $1 \mathrm{M}$.

Figure 3. In vitro activity analysis of putative yeast PPCDCs allows the proposal of a model for functional S. cerevisiae PPCDC. (a) PPCDC activity (based on product (3) formation) of $0.5 \mu \mathrm{g}$ each of Hal3 $(\mathbf{H})$, Vhs3 (V), Ykl088w (Y) individually and in various combinations as 
indicated. The data shown are the average of three replicates, are corrected for an average measured background rate and were normalized to the activity of the Hal3/Vhs3/Ykl088w mixture $(0.5 \mu \mathrm{g}$ of each), which is roughly equivalent to the activity of $\sim 0.73 \mu \mathrm{g}$ (30 pmol) HsCoaC used as positive control. Error bars denote s.d. (b) A cartoon of the homotrimeric A. thaliana PPCDC enzyme (AtHal3a), showing the active sites containing the conserved His and Cys residues at the interfaces of the protomers. (c) A cartoon of the proposed heterotrimeric structure of a potentially active $S$. cerevisiae PPCDC enzyme consisting of one protomer each of Hal3, Vhs3 and Ykl088w, with the predicted single functional active site highlighted. A model in which the Hal3 and Vhs3 protomers are interchanged should give a similarly active enzyme.

Figure 4. The yeast PPCDC is a trimer consisting of Hal3, Vhs3 and Ykl088w. (a) SDSPAGE analysis of Hal3 and Vhs3 pulled-down with immobilized Ykl088w, followed by cross-linking: (1) Hal3; (2) Vhs3; (3) GST-Ykl088w-containing beads; (4) sample 3 incubated with purified Hal3 and Vhs3; (5) material eluted from sample 4 after cleavage of Ykl088w from beads; (6) sample 5 cross-linked with $0.005 \%$ glutaraldehyde ( $2 \times$ the amount loaded); (7) $2 \mu \mathrm{g}$ BSA; (8) $2 \mu \mathrm{g}$ BSA after cross-linking. The $~ 70 \mathrm{kDa}$ protein present in all recombinant yeast samples is probably of E. coli origin. (b) Stoichiometric activity analysis of binary mixtures of Hal3 $(\mathbf{H})$ and Ykl088w (Y) relative to the ternary Hal3/Vhs3/Ykl088w combination (values indicate each protein's quantity in $\mu \mathrm{g}$ ). The H:0.5,Y:0.5 sample’s activity is significantly different from those of the two samples with increased amounts of either $\mathbf{H}$ or $\mathbf{Y}(\mathrm{P}<0.05$, unpaired t-test assuming equal variance). The data are the average of two replicates (corrected and normalized as in Figure 3a), with error bars denoting s.d. (c) Activity analysis based on product formation (closed bars) and $\mathrm{CO}_{2}$ release (open bars) of Ykl088w-containing native combinations, compared to the same combinations in which conserved His or Cys residues have been mutated. H*, Hal3 H378A; V*, Vhs3 H459A; Y*, Ykl088w C478S. The data are the average of three replicates (corrected and normalized as in Figure 3a), with error bars denoting s.d. Asterisks above the bars indicate the confidence level in the values being significantly different from zero (*, $\mathrm{P}<0.05$; **, $\mathrm{P}<0.01$ ). N.d., not determined.

Figure 5. Replacement of the sequence of Ykl088w's motif II with the sequence of the same motif from Hal3 results in a functional PPCDC protein. (a) Alignment of residues surrounding AtHal3a’s conserved His90 and the equivalent regions of Hal3, Vhs3 and 
Ykl088w. Shaded sequences indicate the Hal3 sequence engineered to replace equivalent Ykl088w sequence. The conserved His is enlarged and other conserved residues are denoted in boldface. (b) E. coli strain BW369 $\left(d f p-707^{\text {ts }}\right)$, which carries a mutation that abolishes PPCDC activity at $37^{\circ} \mathrm{C}$, was transformed with the indicated plasmids expressing the human PPCDC (pPROEX-HsCoaC), all three native yeast proteins and reconstructed YKL088w_His ${ }^{\text {Rep }}$. Transformants were grown at 30 and $37^{\circ} \mathrm{C}$ (permissive and nonpermissive temperatures, respectively). (c) PPCDC activity (based on product formation) of the engineered homomeric Ykl088wHis ${ }^{\text {Rep }}$ protein $\left(\mathbf{Y}^{\mathbf{H R}}\right)$ present in various amounts (in $\left.\mu \mathrm{g}\right)$ as indicated, and in combination with Hal3 and Vhs3. Hal3 (3.0 $\mu \mathrm{g})$, Vhs3 (3.0 $\mu \mathrm{g})$ and Yk1088w (1.5 $\mu \mathrm{g})$ were used as negative controls; only Hal3 is shown. The data are the average of three replicates (corrected and normalized as in Figure 3a), with error bars denoting s.d. Asterisks above the bars indicate the confidence level in the values being significantly different from zero $(*, \mathrm{P}<0.05$; **, $\mathrm{P}<0.01)$.

Figure 6. The divergence of PPCDC monomers in yeasts. Forty-three protein sequences of putative yeasts PPCDC were aligned using the ClustalW2 algorithm at the EMBL-EBI server and a phylogenetic tree was generated (iteration parameter was set to "tree" and clustering was performed by the Neighbour-Joining method). The unrooted tree was draw with the Phylodraw software ${ }^{50}$ and labels were added manually. S. cerevisiae proteins are denoted in boldface. Shaded groups (1 and 2) correspond to proteins found in Hemiascomycetous yeast. See main text for details. 


\section{Reference List}

1. Begley,T.P., Kinsland,C., \& Strauss,E. The biosynthesis of coenzyme A in bacteria. Vitam. Horm. 61, 157-171 (2001).

2. Mercer,A.C. \& Burkart,M.D. The ubiquitous carrier protein--a window to metabolite biosynthesis. Nat. Prod. Rep. 24, 750-773 (2007).

3. Daugherty,M. et al. Complete reconstitution of the human coenzyme A biosynthetic pathway via comparative genomics. J. Biol. Chem. 277, 21431-21439 (2002).

4. Kupke,T., Hernandez-Acosta,P., \& Culianez-Macia,F.A. 4'-phosphopantetheine and coenzyme A biosynthesis in plants. J. Biol. Chem. 278, 38229-38237 (2003).

5. Ye,Y., Osterman,A., Overbeek,R., \& Godzik,A. Automatic detection of subsystem/pathway variants in genome analysis. Bioinformatics. 21 Suppl 1, i478-i486 (2005).

6. Osterman,A. \& Overbeek,R. Missing genes in metabolic pathways: a comparative genomics approach. Curr. Opin. Chem. Biol. 7, 238-251 (2003).

7. Kupke,T., Hernandez-Acosta,P., Steinbacher,S., \& Culianez-Macia,F.A. Arabidopsis thaliana flavoprotein AtHAL3a catalyzes the decarboxylation of 4'-Phosphopantothenoylcysteine to 4'phosphopantetheine, a key step in coenzyme A biosynthesis. J. Biol. Chem. 276, 19190-19196 (2001).

8. Strauss,E., Kinsland,C., Ge,Y., McLafferty,F.W., \& Begley,T.P. Phosphopantothenoylcysteine synthetase from Escherichia coli. Identification and characterization of the last unidentified coenzyme A biosynthetic enzyme in bacteria. J. Biol. Chem. 276, 13513-13516 (2001).

9. Hernandez-Acosta,P., Schmid,D.G., Jung,G., Culianez-Macia,F.A., \& Kupke,T. Molecular characterization of the Arabidopsis thaliana flavoprotein AtHAL3a reveals the general reaction mechanism of 4'-phosphopantothenoylcysteine decarboxylases. J. Biol. Chem. 277, 2049020498 (2002).

10. Steinbacher,S. et al. Crystal structure of the plant PPC decarboxylase AtHAL3a complexed with an ene-thiol reaction intermediate. J. Mol. Biol. 327, 193-202 (2003).

11. Strauss,E., Zhai,H., Brand,L.A., McLafferty,F.W., \& Begley,T.P. Mechanistic studies on phosphopantothenoylcysteine decarboxylase: trapping of an enethiolate intermediate with a mechanism-based inactivating agent. Biochemistry 43, 15520-15533 (2004).

12. Ferrando,A., Kron,S.J., Rios,G., Fink,G.R., \& Serrano,R. Regulation of cation transport in Saccharomyces cerevisiae by the salt tolerance gene HAL3. Mol. Cell Biol. 15, 5470-5481 (1995).

13. Di Como,C.J., Bose,R., \& Arndt,K.T. Overexpression of SIS2, which contains an extremely acidic region, increases the expression of SWI4, CLN1 and CLN2 in sit4 mutants. Genetics 139, 95-107 (1995).

14. Arino,J. Novel protein phosphatases in yeast. Eur. J. Biochem. 269, 1072-1077 (2002).

15. de Nadal,E. et al. The yeast halotolerance determinant Hal3p is an inhibitory subunit of the Ppz1p Ser/Thr protein phosphatase. Proc. Natl. Acad. Sci. U. S. A 95, 7357-7362 (1998).

16. Posas,F., Camps,M., \& Arino,J. The PPZ protein phosphatases are important determinants of salt tolerance in yeast cells. J. Biol. Chem. 270, 13036-13041 (1995). 
17. Yenush,L., Mulet,J.M., Arino,J., \& Serrano,R. The Ppz protein phosphatases are key regulators of $\mathrm{K}+$ and $\mathrm{pH}$ homeostasis: implications for salt tolerance, cell wall integrity and cell cycle progression. EMBO J. 21, 920-929 (2002).

18. Ruiz,A., Yenush,L., \& Arino,J. Regulation of ENA1 Na(+)-ATPase gene expression by the Ppz1 protein phosphatase is mediated by the calcineurin pathway. Eukaryot. Cell 2, 937-948 (2003).

19. Lee,K.S., Hines,L.K., \& Levin,D.E. A pair of functionally redundant yeast genes (PPZ1 and PPZ2) encoding type 1-related protein phosphatases function within the PKC1-mediated pathway. Mol. Cell Biol. 13, 5843-5853 (1993).

20. Merchan,S., Bernal,D., Serrano,R., \& Yenush,L. Response of the Saccharomyces cerevisiae Mpk1 mitogen-activated protein kinase pathway to increases in internal turgor pressure caused by loss of Ppz protein phosphatases. Eukaryot. Cell 3, 100-107 (2004).

21. Clotet,J., Gari,E., Aldea,M., \& Arino,J. The yeast ser/thr phosphatases sit4 and ppz1 play opposite roles in regulation of the cell cycle. Mol. Cell Biol. 19, 2408-2415 (1999).

22. Munoz,I., Simon,E., Casals,N., Clotet,J., \& Arino,J. Identification of multicopy suppressors of cell cycle arrest at the G1-S transition in Saccharomyces cerevisiae. Yeast 20, 157-169 (2003).

23. Ruiz,A. et al. Functional characterization of the Saccharomyces cerevisiae VHS3 gene: a regulatory subunit of the Ppz1 protein phosphatase with novel, phosphatase-unrelated functions. J. Biol. Chem. 279, 34421-34430 (2004).

24. Giaever,G. et al. Functional profiling of the Saccharomyces cerevisiae genome. Nature 418, 387-391 (2002).

25. Espinosa-Ruiz,A., Belles,J.M., Serrano,R., \& Culianez-Macia,F.A. Arabidopsis thaliana AtHAL3: a flavoprotein related to salt and osmotic tolerance and plant growth. Plant J. 20, 529539 (1999).

26. Albert,A. et al. The X-ray structure of the FMN-binding protein AtHal3 provides the structural basis for the activity of a regulatory subunit involved in signal transduction. Structure. Fold. Des 8, 961-969 (2000).

27. Strauss,E. \& Begley,T.P. Mechanistic studies on phosphopantothenoylcysteine decarboxylase. J. Am. Chem. Soc. 123, 6449-6450 (2001).

28. Strauss,E. \& Begley,T.P. Stereochemical studies on phosphopantothenoylcysteine decarboxylase from Escherichia coli. Bioorg. Med. Chem. Lett. 13, 339-342 (2003).

29. Kupke,T. \& Schwarz,W. 4'-phosphopantetheine biosynthesis in Archaea. J. Biol. Chem. 281, 5435-5444 (2006).

30. de Nadal,E., Fadden,R.P., Ruiz,A., Haystead,T., \& Arino,J. A role for the Ppz Ser/Thr protein phosphatases in the regulation of translation elongation factor 1Balpha. J. Biol. Chem. 276, 14829-14834 (2001).

31. Costigan,C., Kolodrubetz,D., \& Snyder,M. NHP6A and NHP6B, which encode HMG1-like proteins, are candidates for downstream components of the yeast SLT2 mitogen-activated protein kinase pathway. Mol. Cell Biol. 14, 2391-2403 (1994).

32. Gavin,A.C. et al. Proteome survey reveals modularity of the yeast cell machinery. Nature $\mathbf{4 4 0}$, 631-636 (2006). 
33. Brown,M.T., Goetsch,L., \& Hartwell,L.H. MIF2 is required for mitotic spindle integrity during anaphase spindle elongation in Saccharomyces cerevisiae. J. Cell Biol. 123, 387-403 (1993).

34. Kupke,T. et al. Molecular characterization of lantibiotic-synthesizing enzyme EpiD reveals a function for bacterial Dfp proteins in coenzyme A biosynthesis. J. Biol. Chem. 275, 3183831846 (2000).

35. Hazbun,T.R. et al. Assigning function to yeast proteins by integration of technologies. Mol. Cell 12, 1353-1365 (2003).

36. Krogan,N.J. et al. Global landscape of protein complexes in the yeast Saccharomyces cerevisiae. Nature 440, 637-643 (2006).

37. Collins,S.R. et al. Toward a comprehensive atlas of the physical interactome of Saccharomyces cerevisiae. Mol. Cell Proteomics. 6, 439-450 (2007).

38. Spitzer,E.D. \& Weiss,B. dfp Gene of Escherichia coli K-12, a locus affecting DNA synthesis, codes for a flavoprotein. J. Bacteriol. 164, 994-1003 (1985).

39. Spitzer,E.D., Jimenez-Billini,H.E., \& Weiss,B. beta-Alanine auxotrophy associated with dfp, a locus affecting DNA synthesis in Escherichia coli. J. Bacteriol. 170, 872-876 (1988).

40. Dujon,B. Yeasts illustrate the molecular mechanisms of eukaryotic genome evolution. Trends Genet. 22, 375-387 (2006).

41. Munoz,I. et al. Functional characterization of the yeast Ppz1 phosphatase inhibitory subunit Hal3: a mutagenesis study. J. Biol. Chem. 279, 42619-42627 (2004).

42. Bucovaz,E.T., Macleod,R.M., Morrison,J.C., \& Whybrew,W.D. The coenzyme A-synthesizing protein complex and its proposed role in CoA biosynthesis in bakers' yeast. Biochimie 79, 787798 (1997).

43. Bucovaz,E.T. et al. Coenzyme A-synthesizing protein complex of Saccharomyces cerevisiae. Mol. Cell Biochem. 30, 7-26 (1980).

44. Olzhausen,J., Schubbe,S., \& Schuller,H.J. Genetic analysis of coenzyme A biosynthesis in the yeast Saccharomyces cerevisiae: identification of a conditional mutation in the pantothenate kinase gene CAB1. Curr. Genet. 55, 163-173 (2009).

45. Gancedo,C. \& Flores,C.L. Moonlighting proteins in yeasts. Microbiol. Mol. Biol. Rev. 72, 197210 (2008).

46. Adams,A., Gottschling,D.E., Kaiser,C.A., \& Stearns,T. Methods in Yeast Genetics(Cold Spring Harbor Laboratory Press, NY,1997).

47. Treco,D.A. \& Winston,F. Current Protocols in Molecular Biology (eds. Ausubel,F.M. et al.) 13.2.10-13.2.12 (John Wiley \& Sons, NY, 1998).

48. Garcia-Gimeno,M.A., Munoz,I., Arino,J., \& Sanz,P. Molecular Characterization of Ypi1, a Novel Saccharomyces cerevisiae Type 1 Protein Phosphatase Inhibitor. J. Biol. Chem. 278, 47744-47752 (2003).

49. Meyer,C.R., Rustin,P., \& Wedding,R.T. A Simple and Accurate Spectrophotometric Assay for Phosphoenolpyruvate Carboxylase Activity. Plant Physiol 86, 325-328 (1988). 
50. Choi,J.H., Jung,H.Y., Kim,H.S., \& Cho,H.G. PhyloDraw: a phylogenetic tree drawing system. Bioinformatics. 16, 1056-1058 (2000). 
Table 1. The diploid strains MAR25 (ykl088w/YKL088w), AGS4 (hal3/HAL3 vhs3/VHS3) and AGS31 (ykl088w/YKL088w hal3/HAL3 vhs3/VHS3) were transformed with plasmids bearing wild type or mutated versions of $S$. cerevisiae HAL3, VHS3 and YKL088w, as well as plant (AtHal3a), mouse (MmCoaC), human (HsCoaC) and E. coli (dfp) PPCDCs. The ability to recover viable cells was monitored by a combination of tetrad analysis and/or random spore analysis. The $(+)$ symbol indicates that expression of the gene rescues the lethal phenotype in haploid derivatives, whereas (-) denotes the inability to do so. n.d., not determined.

\begin{tabular}{|c|c|c|c|c|}
\hline \multirow[b]{2}{*}{ Gene } & \multirow[b]{2}{*}{ Version } & \multicolumn{3}{|c|}{ Heterozygous diploid strain } \\
\hline & & hal3 vhs3 & $y k l 088 w$ & $\begin{array}{c}\text { hal3 vhs3 } \\
\text { ykl088w }\end{array}$ \\
\hline HAL3 & WT & + & - & n.d. \\
\hline VHS3 & WT & + & - & n.d. \\
\hline YKL088w & WT & - & + & n.d. \\
\hline AtHal3a & WT & + & + & + \\
\hline MmCoaC & WT & + & + & + \\
\hline HsCoaC & WT & + & + & + \\
\hline E. coli $d f p$ & WT & + & + & + \\
\hline HAL3 & H378A & - & n.d. & n.d. \\
\hline VHS3 & H459A & - & n.d. & n.d. \\
\hline YKL088w & H391A & n.d. & + & n.d. \\
\hline YKL088w & C478S & n.d. & - & n.d. \\
\hline AtHal3a & H90A & - & + & - \\
\hline AtHal3a & C175S & + & - & - \\
\hline HsCoaC & C175S & + & - & - \\
\hline
\end{tabular}




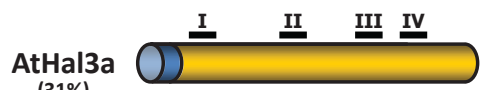

(31\%)
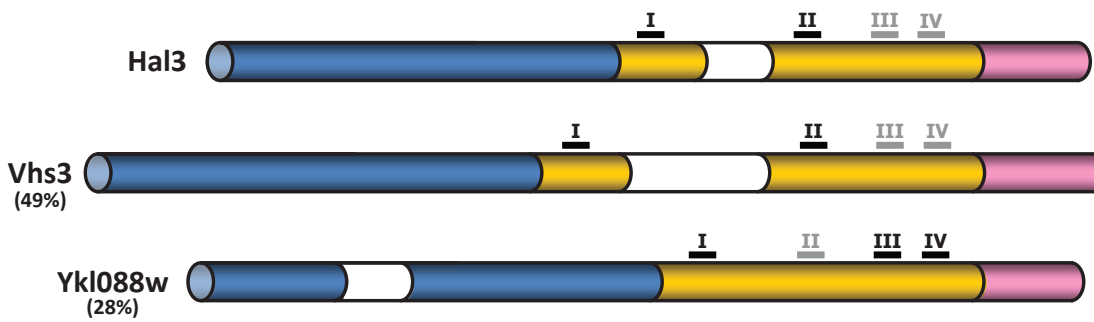

\section{b}

AtHal3a

Hal3

Vhs 3

Yk1088w
20

255

283

293

I

RV-------------LLAASGSVAAIKFGNLCHCFTEWA-----EVRAVVTKSS LHFLDK-------
RLP-------QDDGKLHVLFGATGSLSVFKIKPMIKKLEEIYGRDRISIQVILTQSATQFFEQ-INSERT-

RV--------------LLAASGSVAAIKFGNLCHCFTEWA-----EVRAVVTKS S LHFLDK-------

PRLP------QDDGKLHVLFGATGSLSVFKLKHMIRKLEEIYGRDKICIQVILTNSATKFFAM-INSERT-

II

AtHal3a

Hal3

Vhs 3

Yk1088w

62 LSLPQEVTLYTDEDEWS--SWNKIG-----DPVLHIELRRWADVLVIAPLSANTLGKIAGGLCDNLLTCIIR

350 VELPPHIQLWTDQDEWD--AWKQRT-----DPVLHIELRRWADILVVAPLTANTLSKIALGLCDNLLTSVIR

431 IELPAHIQFWTDQDEWD--VWRQRT-----DPVLHIELRRWADILVVAPLTANTLAKIALGLCDNLLTSVIR

356 LKMSTHVKIWREEDAWVFDAVNKNDTSLSLNL I LHHELRKWADI FLIAPLSANTLAKLANG ICNNLLTSVMR

\section{III}

*

\section{IV}

AtHal3a 127 AWDYTKPLFVAPAMNTLMWNNPFTERHLLSLDELG--ITLIPPIKKRL-ACGDYGNGAMAEPSLIYSTVRLF Hal3

Vhs 3

Yk1088w

415 AWNPSYPILLAPSMVSSTFNSMMTKKQLQTIKEEMSWVTVEKPSEKVMDINGDIGLGGMMDWNEIVNKIVMK

496 AWNPTFPIFLAPSMGSGTFNS IMTKKHFRI IQEEMPWVTVFKPSEKVMGINGDIGLSGMMDANEIVGKIVVK

428 DWSPLTPVLIAPAMNTFMYINPMTKKHLTSLVQDYPFIQVLKPVEKVL-ICGDIGMGGMREWTDIVEIVRRR

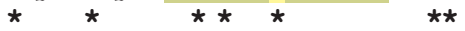

C<smiles>CC(C)(COP(=O)(O)O)[C@@H](O)C(=O)NCCC(=O)N[C@@H](CS)C(=O)O</smiles>

4'-Phosphopantothenoylcysteine (PPC; 2)<smiles>CC(C)(COP(=O)(O)O)[C@@H](O)C(=O)NCCC(=O)NCCS</smiles>

4'-Phosphopantetheine (PP; 3)
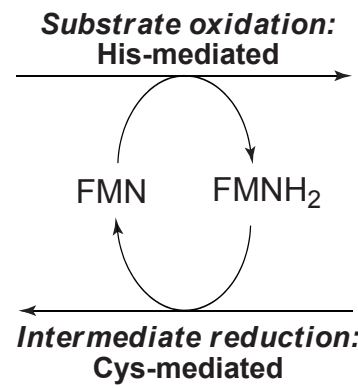

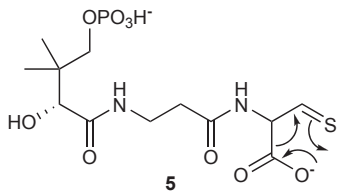<smiles></smiles> 
Strain

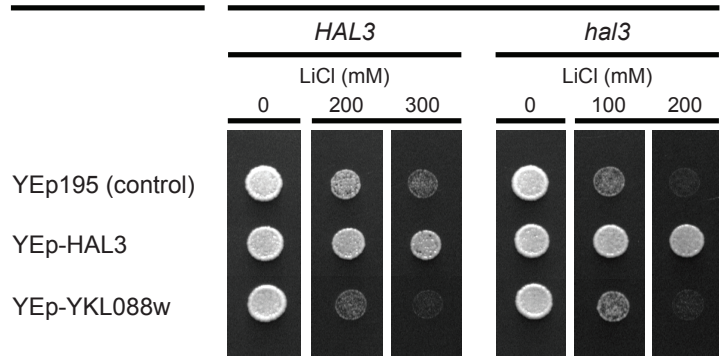

b

Plasmid

Strain

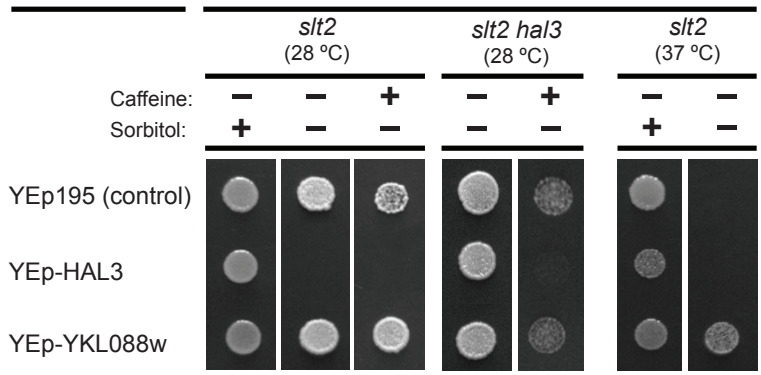




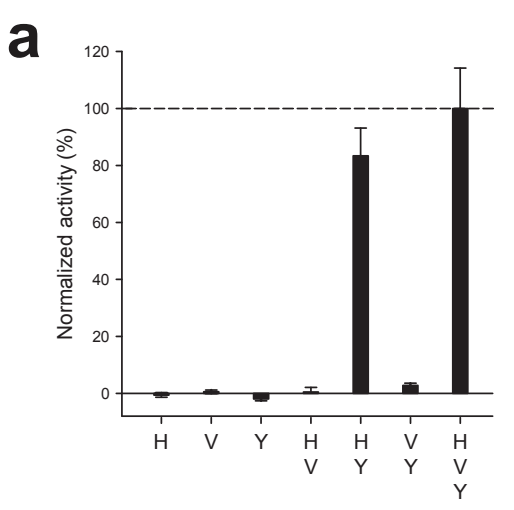

b

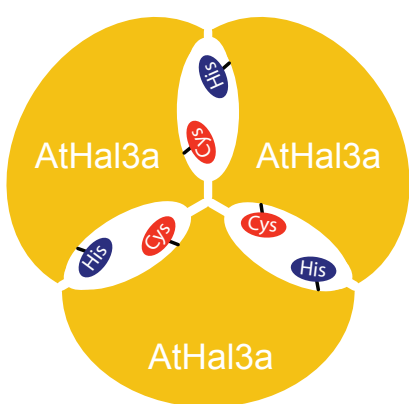

A. thaliana
C single functional active site

Ykl088w

S. cerevisiae 
$\mathbf{a}_{\text {tos }} \quad \mathbf{b}$

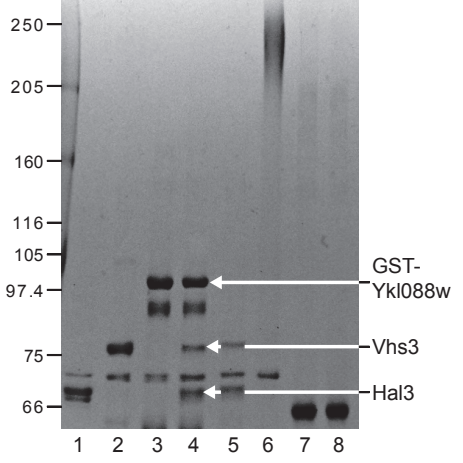

C

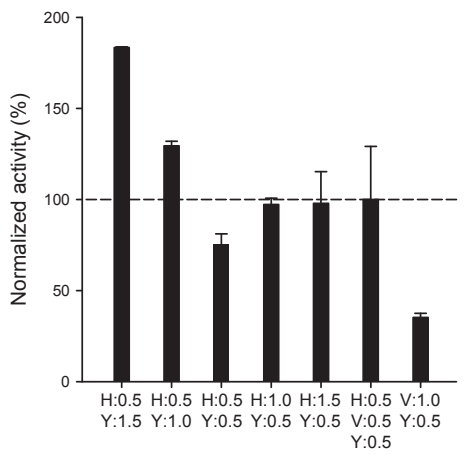

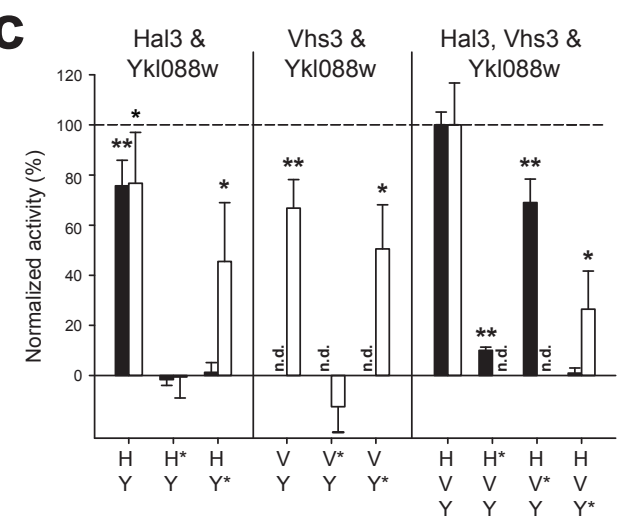


a AtHal3a 77 EWS--SWNKIG-----DPVLHIELRRWAD 98 ScHal3 365 EWD--AWKQRT----DPVLHIELRRWAD 386 Vhs3 466 EWD--VWRQRT-----DPVLHIELRRWAD 467 Yk1088w 371 AWVFDAVNKNDTSLSLNLILHHELRKWAD 399

b

pPROEX-HsCoaC

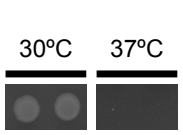

C 100

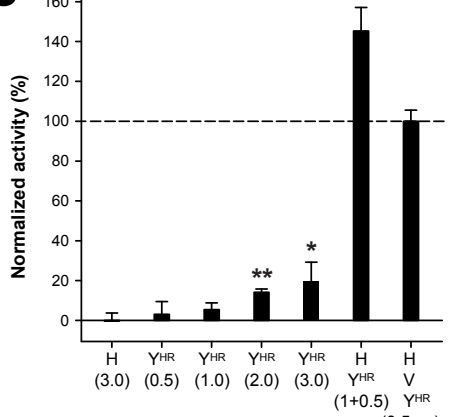

pGEX-HAL3

pGEX-VHS3
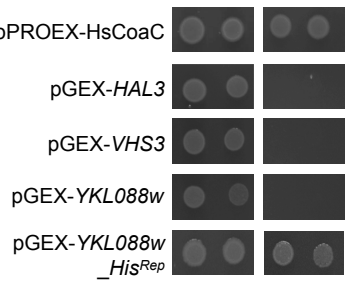

(0.5 ea) 


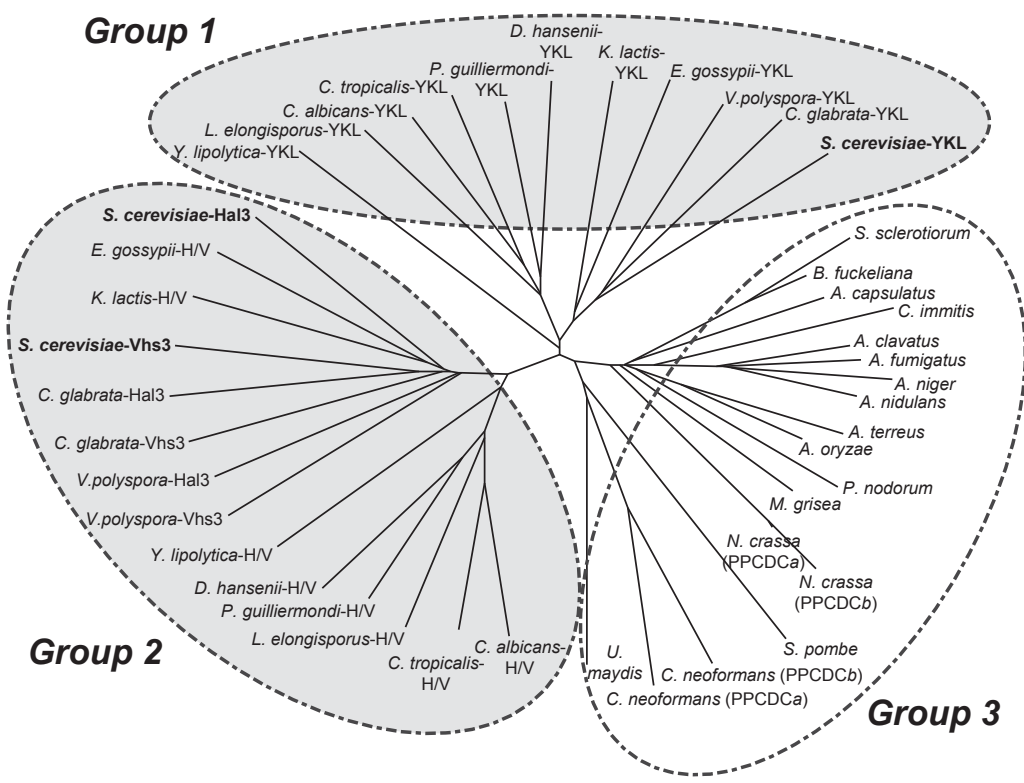

